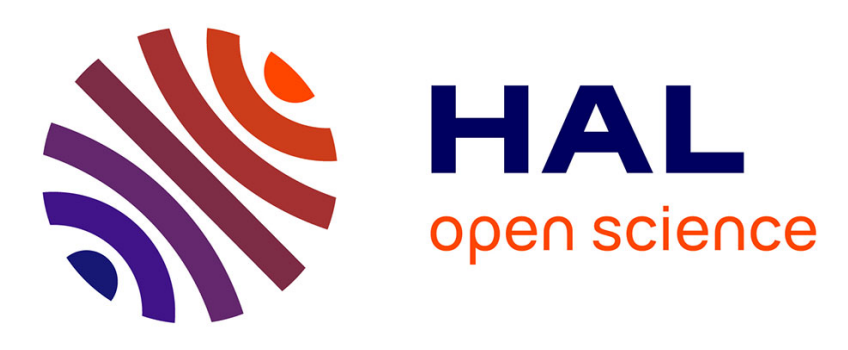

\title{
Enjeux psychosociaux du don de sperme: le point de vue des couples.
}

\author{
Nikos Kalampalikis, Valérie Haas, Nicolas Fieulaine, Marjolaine Doumergue, \\ G. Deschamps, H. Dupont
}

\section{- To cite this version:}

Nikos Kalampalikis, Valérie Haas, Nicolas Fieulaine, Marjolaine Doumergue, G. Deschamps, et al.. Enjeux psychosociaux du don de sperme: le point de vue des couples.. In P. Jouannet \& R. Mieusset (Eds.), Donner et après. La procréation par don de spermatozoïdes avec ou sans anonymat?. Donner et après. La procréation par don de spermatozoïdes avec ou sans anonymat?, Springer, pp. 55-68, 2010, 978-2-8178-0121-6. halshs-00931450

\section{HAL Id: halshs-00931450 \\ https://shs.hal.science/halshs-00931450}

Submitted on 19 Dec 2018

HAL is a multi-disciplinary open access archive for the deposit and dissemination of scientific research documents, whether they are published or not. The documents may come from teaching and research institutions in France or abroad, or from public or private research centers.
L'archive ouverte pluridisciplinaire HAL, est destinée au dépôt et à la diffusion de documents scientifiques de niveau recherche, publiés ou non, émanant des établissements d'enseignement et de recherche français ou étrangers, des laboratoires publics ou privés. 


\title{
Enjeux psychosociaux du don de sperme : le point de vue des couples
}

\author{
N. Kalampalikis, V. Haas, N. Fieulaine, M. Doumergue, \\ G. Deschamps et H. Chiron
}

De nos jours, de plus en plus de couples rencontrant des difficultés de procréation, notamment d'infertilité masculine et/ou de stérilité, font appel à l'insémination artificielle avec tiers donneur (IAD) qui a permis la naissance d'environ 50000 enfants jusqu'à présent en France [1]. En outre, on observe une tendance croissante de couples ayant déjà eu un enfant par IAD qui en souhaitent un deuxième, voire un troisième. Les CECOS (centres d'étude et de conservation des œufs et du sperme humains), depuis 1973, assurent la congélation et la conservation des spermatozoïdes, garantissent l'anonymat et la gratuité du don, tandis que leur fonctionnement est réglementé par les lois françaises de bioéthique. Grâce à un partenariat de recherche établi avec la Fédération française des CECOS, nous avons eu la rare possibilité d'avoir accès à un échantillon large de la population impliquée dans l'acte du don de sperme (membres de couples demandeurs, $n=459$; donneurs $n=33$ ), complété par l'échantillon stratifié d'une population non impliquée dans les procréations médicalement assistées $(n=598)$, nous permettant de réaliser une étude nationale sur les enjeux psychosociaux du don de sperme de 2006 à 2008 [2]. Dans le cadre de cet article, nous nous focaliserons uniquement sur certaines prises de position des couples demandeurs-receveurs interrogés par questionnaire à l'égard de la démarche du don, ses principes et ses enjeux identitaires et psychosociaux.

\section{Construction d'un outil adapté}

La construction du questionnaire à destination des couples est le fruit d'une triangulation méthodologique [3]. Tout d'abord, les données recueillies durant les phases exploratoires et qualitatives nous ont permis de disposer d'une première approche des enjeux posés par la démarche d'IAD, et ce, sous différents angles, celui du grand public, celui des donneurs et celui des couples demandeurs. Nous avons également appuyé l'élaboration de l'outil quantitatif sur la 
littérature scientifique internationale existante qui, pour une part des travaux, utilisait ce type de méthodologie et qui, pour une autre part, venait compléter avantageusement les observations réalisées dans le cadre de cette recherche.

À ce titre, le questionnaire élaboré, ainsi que les données qu'il a permis de recueillir ne peuvent être envisagés de manière isolée ou autosuffisante. Pensée comme le prolongement d'une exploration qualitative en profondeur des enjeux symboliques et identitaires soulevés par l'IAD, et comme un moyen pertinent de compléter et d'approfondir les constats établis en réponse à une demande, la démarche quantitative présentée ici n'a de sens que mise en regard des autres données recueillies. Elle n'est en aucun cas une confirmation ou une infirmation des observations précédentes, mais un angle supplémentaire d'investigation permettant des constats systématiques et comparatifs chiffrés, dont la validité repose essentiellement sur son articulation aux autres phases de l'étude.

Les questions de l'anonymat et de la gratuité s'ancrent dans un "halo» de significations plus larges, renvoyant à des interrogations sociales et anthropologiques qui concernent la famille, la parentalité, la normalité, la nature ou encore le don [4-6]. L'analyse des positions sur l'anonymat et la confidentialité ne peut donc se réduire au recueil d'opinions sur ces seules questions, sinon en courant le risque de réduire la complexité des problématiques en jeu à leur expression simpliste et contrainte par le cadre de l'enquête ou plutôt du sondage.

Les principaux thèmes du questionnaire ont été abordés de manière à pouvoir établir leurs relations dynamiques et le rôle joué par ces relations dans les positionnements individuels. La famille et sa dimension normative, la stérilité, le donneur et le don, la parentalité et la gestion du secret, l'anonymat et la gratuité sont autant de thèmes repérés comme saillants. L'hypothèse qui a présidé à l'élaboration de l'outil quantitatif est celle d'une interdépendance de ces différents aspects de la problématique de l'IAD et du caractère déterminant de cette interdépendance dans les prises de position à l'égard de questions spécifiques telles que le secret, l'anonymat ou la gratuité. Ce souci d'explorer le plus complètement possible les configurations de sens relatives à l'IAD, en adoptant une méthodologie propice à faire apparaître également des contradictions ou des ambiguïtés (le niveau d'accord plutôt que les choix multiples permet l'expression de telles contradictions par l'accord pour deux items aux sens opposés), a été complété d'une volonté d'assurer autant que possible la comparabilité des données recueillies aux recherches existantes. Dans ce sens, nous avons intégré à ce questionnaire différents items déjà utilisés dans la littérature [7-11], exclusivement anglo-saxonne, étant donné qu'aucune enquête de ce type n’avait encore été réalisée dans le contexte français. 


\section{Population et contexte sensibles de recueil des données}

Après une phase de prétest concluante auprès d'une dizaine de couples dans des conditions identiques à celles préconisées, les passations du questionnaire se sont déroulées durant cinq mois, de la mi-juin à la mi-novembre 2008. Le partenariat avec la Fédération des CECOS nous a permis de cibler tous les centres. Le recrutement des sujets a été assuré aussi bien par notre équipe que par des professionnels des CECOS disposant de consignes précises fournies par nos soins. La consigne introductive du questionnaire présentait le cadre académique de la recherche et invitait les sujets à participer anonymement à « une étude relative aux questions posées par la technique de l'insémination artificielle avec donneur ». La passation du questionnaire était individuelle et a été réalisée sur place aux CECOS en présence d'un professionnel du centre ou d'un chercheur. Une codification a posteriori permettait d'identifier les questionnaires issus des membres d'un même couple pour des analyses ultérieures, longitudinales et comparatives. Il mérite de rappeler ici que la particularité du profil de notre population, comme pour l'échantillon des donneurs, a restreint la passation du questionnaire uniquement aux couples présents dans les différents CECOS au moment de notre recherche. Cette variable contextuelle n'est pas sans incidence quant au mode concret de passation ( «forcément » sur place aux CECOS lors d'une visite en rapport avec leur dossier), et à l'état d'esprit des répondants ( forcément» pris dans la temporalité d'une demande qui leur est capitale). Au final, 459 questionnaires (taux de participation $75 \%$ ) ont été recueillis auprès de 20 CECOS ayant accepté de participer à la collecte des données réparties dans tout le territoire.

\section{Profil sociodémographique des couples interrogés}

Sur les 459 questionnaires recueillis, 49 sont arrivés hors délais et huit se sont révélés inexploitables. L’analyse présentée ici a porté sur 407 questionnaires issus de 201 couples (cinq sujets n'ayant pas donné d'information de genre). Le tableau ci-après, qui récapitule les caractéristiques sociodémographiques de l'échantillon, laisse apparaître que celui-ci est constitué de couples d'âge moyen, dont la répartition en termes de niveaux de diplômes et de catégories socioprofessionnelles est relativement équilibrée, et pour laquelle l'on constate des différences classiquement observées entre hommes et femmes, les secondes étant davantage diplômées mais davantage touchées par le chômage et occupant des emplois plus faiblement rémunérés. On constate par ailleurs que les personnes inactives (en particulier les femmes au foyer) ou au chômage sont largement minoritaires, la quasi-totalité des personnes interrogées déclarant avoir actuellement un emploi (tableau I). 
Tableau I. - Caractéristiques sociodémographiques des couples.

\begin{tabular}{|l|c|c|c|}
\hline & Total & Hommes & Femmes \\
\hline Effectif & 402 & $201(50 \%)$ & $201(50 \%)$ \\
\hline Âge $(\mathrm{m}, \mathrm{SD})$ & $33,1(5,46)$ & $31,2(4,73)$ & $35(5,97)$ \\
\hline Lieu de naissance & & & \\
\hline France & $91,1 \%$ & $93,5 \%$ & $91 \%$ \\
\hline Hors-France & $7 \%$ & $6 \%$ & $7,9 \%$ \\
\hline Diplôme & & & \\
\hline Inférieur au bac & $37,7 \%$ & $46,8 \%$ & $28,6 \%$ \\
\hline Bac & $17,5 \%$ & $16,4 \%$ & $18,6 \%$ \\
\hline Supérieur au bac & $44,7 \%$ & $36,8 \%$ & $52,8 \%$ \\
\hline Situation & & & $2,7 \%$ \\
\hline Inactif & $2,4 \%$ & $2,2 \%$ & $8,5 \%$ \\
\hline Au chômage & $6 \%$ & $3,3 \%$ & $88,8 \%$ \\
\hline Travail & $91,6 \%$ & $94,5 \%$ & \\
\hline CSP & & & $26,3 \%$ \\
\hline Supérieure & $30,1 \%$ & $33,7 \%$ & $13,7 \%$ \\
\hline Intermédiaire & $14,6 \%$ & $15,5 \%$ & $58,3 \%$ \\
\hline Inférieure & $52 \%$ & $46 \%$ & $1,7 \%$ \\
\hline Autres & $3,3 \%$ & $4,8 \%$ & \\
\hline
\end{tabular}

CSP : catégories socio-professionnelle.

L'échantillon analysé est parfaitement équilibré en ce qui concerne la répartition par genre, avec $50 \%$ d'hommes et $50 \%$ de femmes. Cependant, les données ne sont pas suffisantes pour pouvoir affirmer que ces hommes et ces femmes sont issus du même couple. Effectivement, certaines personnes ont répondu seules au questionnaire, du fait de l'absence, de l'indisponibilité ou du refus du conjoint. La moyenne d'âge est de 33,1 ans au total, légèrement plus élevée chez les femmes ( 35 ans) que chez les hommes (31,2 ans), et quasiment tous les couples demandeurs (91,1\%) sont nés en France.

\section{Éléments sur les contextes de la démarche}

Afin de contextualiser les réponses obtenues au questionnaire et ainsi mieux saisir les caractéristiques du "moment » où les répondants ont été sollicités, il nous semble nécessaire de présenter au préalable différents aspects de leurs situations vis-à-vis de la démarche d'IAD. Tout d'abord, les couples interrogés se déclarent majoritairement mariés ou en concubinage $(87 \%)$, et de manière marginale en union libre ou pacsés $(11,5 \%)$. Le taux de mariage est de 65,6\%. Ensuite, seule une minorité a déjà un - ou des - enfant(s) conçus hors IAD (qu'il s'agisse de conceptions sans PMA, ou recourant à d'autres techniques de PMA que l'IAD). Par ailleurs, il apparaît qu'environ un quart 
des couples (23\%) a déjà au moins un enfant conçu par IAD, et que donc $75 \%$ des couples interrogés sont dans leur première démarche d'IAD (ce qui correspond aux statistiques nationales sur les couples demandeurs, qui font apparaître une répartition stable entre premières demandes et deuxièmes ou plus - respectivement 75 et $25 \%$ ). La majorité de notre échantillon se trouve dans une phase d'attente se situant entre 10 et 24 mois, tandis que le rapport globalement entretenu à la démarche apparaît largement satisfaisant chez les personnes interrogées, tout comme le «sentiment d'être suffisamment informés " qui n'apparaît manquer que chez une minorité des répondants $(5,5 \%)$. Concernant les sources d'information sur l'IAD, elles apparaissent diversifiées, et les différents supports sont considérés de manière variable entre hommes et femmes. Les sources institutionnelles et médicales (CECOS, médecins) apparaissent de loin comme sources principales d'information indépendamment du genre. Les médias, type télévision et Internet, occupent également une place importante. Une différence notable est à souligner entre hommes et femmes concernant l'usage et la participation aux forums Internet, davantage prononcée chez les femmes.

\section{Vécus, partages, secrets}

Notre approche a consisté à recueillir différents aspects du vécu lié à l'IAD, notamment en termes d'expérience et/ou d'intention de partage social de leur démarche (tableau II).

Tableau II. - Expériences, ressentis, intentions du partage.

\begin{tabular}{|c|c|c|c|c|c|c|c|}
\hline & \multicolumn{2}{|c|}{$\begin{array}{c}\text { Total } \\
\%\end{array}$} & \multicolumn{2}{c|}{$\begin{array}{c}\text { Hommes } \\
\%\end{array}$} & \multicolumn{2}{c|}{$\begin{array}{c}\text { Femmes } \\
\%\end{array}$} \\
\hline J'ai discuté de ma démarche d'IAD avec: & Oui & Non & O & N & O & N \\
\hline mon entourage proche amical & $\mathbf{5 6 , 7}$ & 41,1 & 54,7 & 43,8 & 58,7 & 38,3 \\
\hline mon entourage proche familial & 72,4 & 26,4 & 73,1 & 25,9 & 71,6 & 26,8 \\
\hline avec des personnes dans des forums internet & 12,4 & $\mathbf{8 5 , 3}$ & 7,4 & 90 & 17,4 & 80,6 \\
\hline \multicolumn{2}{|l|}{$\begin{array}{l}\text { Cela m'est arrivé de regretter d'en avoir } \\
\text { parlé }\end{array}$} & 20,2 & 76,4 & 15,4 & 80,6 & 24,9 & 72,1 \\
\hline $\begin{array}{l}\text { À l'avenir, je compte discuter de ma démarche } \\
\text { d'IAD avec: }\end{array}$ & Oui & Non & Oui & Non & Oui & Non \\
\hline $\begin{array}{c}\text { mon entourage proche amical } \\
\text { mon entourage proche familial }\end{array}$ & 52,2 & 43,8 & 50,7 & 45,3 & 53,7 & 42,3 \\
\hline $\begin{array}{l}\text { men des personnes dans des forums internet } \\
\text { avec }\end{array}$ & 17,65 & 81,5 & 11,9 & 87,5 & 23,4 & 75,5 \\
\hline
\end{tabular}


La majorité des personnes ont discuté de leur démarche, principalement avec leur famille $(72,4 \%)$ mais aussi avec leurs amis, pour un peu plus de la moitié d'entre eux $(56,7 \%)$. La cellule de l'entourage proche constitue une ressource de partage quasi équitable pour les deux genres. Les forums Internet semblent toucher peu de sujets en général, mais malgré tout, ils représentent un média davantage utilisé par les femmes que par les hommes (corroborant ainsi nos résultats sur les sources d'information). Si nous nous concentrons spécifiquement sur les couples ayant un enfant, l'analyse illustre que les femmes étant déjà mères ont plus parlé de leur démarche au sein de leur famille (83,3 \%) que celles n'ayant pas d'enfant $(71,6 \%)$. La concrétisation du projet parental semble donc modifier, chez les femmes, l'attitude du partage vis-à-vis de la famille. Même si la majorité des sujets ne regrettent pas de s'être exprimés sur leur démarche, les couples avec enfant déclarent plus de regrets. La naissance de l'enfant rend peut-être l'impact de l'annonce plus important. De plus, la seule différence est l'annonce à la famille, plus importante chez les femmes déjà mères, ce qui a pu entraîner des déceptions plus marquées.

En ce qui concerne la question de l'avenir, la majorité des personnes témoignent d'intentions favorables à la perspective du partage, principalement avec la famille. Les forums Internet semblent susciter plus d'intérêt que précédemment, mais restent encore très minoritaires. Les couples avec enfant sont beaucoup moins décidés à s'exprimer sur le sujet, particulièrement auprès de leur entourage amical, pour lequel la majorité des personnes (à 53,7 \%), indépendamment de leur genre, répondent par la négative. Cette différence peut se mettre en lien avec un taux de regret plus important, ce qui conduit ces personnes à réduire la communication sur le sujet autour d'eux. Les regrets pourraient alors concerner l'entourage amical, ce qui expliquerait le refus d'en parler avec celui-ci.

Sur le thème du secret, plusieurs questions visaient à établir les intentions concernant le fait de parler ou non à l'enfant de son mode de conception, mais également les modalités pratiques par lesquelles cette parole peut être envisagée (quand, comment). Le tableau ci-après récapitule les réponses obtenues à ces différentes questions (tableau III).

Les réponses en termes d'intention de parler à l'enfant sont clairement et majoritairement positives et équilibrées par genre. Plus précisément, une majorité de couples se positionnent pour parler à l'enfant de son mode de conception (68\%), des problèmes de fertilité rencontrés $(70 \%)$ et de la démarche d'insémination avec donneur (65\%). Symétriquement, 75,5\% d'entre eux excluent la probabilité de ne rien lui dire et $67,5 \%$ de remettre la question à plus tard.

Néanmoins, même si l'intention d'en parler semble indubitable, le choix du moment paraît plus délicat. Plus de la moitié des couples déclare ne pas savoir exactement quand ils décideront d'en parler. Toutefois, ce choix sera conditionné par une décision des deux parents lorsqu'ils jugeront cela approprié (68\%), sans forcément se fier à la parole ou l'expertise des psychologues (66\%). Un peu plus de la moitié des sujets ( $54 \%$ ) cautionnent implicitement une donnée issue de la recherche qualitative menée auprès d'eux, à savoir l'âge à partir duquel l'enfant s'intéressera lui-même aux questions relatives à la procréation. Enfin, quand 
bien même la moitié des couples interrogés ne sait pas encore "comment» il compte en parler, le mode du récit choisi sera, selon nos sujets, plus proche de la réalité des faits $(72,5 \%)$. Des résultats quasiment identiques ont été constatés auprès de la minorité des couples ayant déjà un enfant né par IAD.

En conclusion, il est possible de dire que les couples souhaitent peu partager leur démarche dans des groupes ou dans un accompagnement, même si les femmes apparaissent systématiquement plus intéressées que les hommes. Les hommes semblent moins prêts à partager leur expérience avec d'autres personnes qu'ils ne connaissent pas (groupe de parole, forum Internet, accompagnement). En revanche, les hommes et les femmes partagent équitablement leur expérience avec leur entourage proche, qu'il soit amical ou familial. De plus, en termes d'intention, hommes et femmes déclarent vouloir partager avec leur enfant à l'avenir le récit "réel » des conditions de sa conception sans pour autant pouvoir définir avec précision le moment adéquat (probablement entre quatre et six ans).

Tableau III. - Parler à l'enfant (mode de conception/modalités).

\begin{tabular}{|l|c|c|}
\hline & \multicolumn{2}{|c|}{ Total } \\
\hline Au sein de notre couple, nous avons décidé de : & $\begin{array}{c}\text { Oui } \\
\%\end{array}$ & $\begin{array}{c}\text { Non } \\
\%\end{array}$ \\
\hline parler à l'enfant de son mode de conception & & \\
\hline parler à l'enfant des problèmes de fertilité rencontrés & $\mathbf{6 8}$ & 27 \\
\hline parler à l'enfant de l'IAD & $\mathbf{7 0}$ & 24 \\
\hline ne rien lui dire & $\mathbf{6 5}$ & 28 \\
\hline remettre cette question à plus tard & 17,5 & $\mathbf{7 5 , 5}$ \\
\hline Si vous comptez en parler à l'enfant, ce sera : & 28 & $\mathbf{6 7 , 5}$ \\
\hline Quand ? & & \\
\hline on ne sait pas encore exactement & $\mathbf{6 0 , 5}$ & 30,5 \\
\hline au moment qui va nous paraitre propice & $\mathbf{6 8}$ & 22,5 \\
\hline au moment que les psychologues considèrent comme propice & 23 & $\mathbf{6 6}$ \\
\hline dès qu'il commencera à poser des questions sur la procréation & $\mathbf{5 4}$ & 35 \\
\hline Comment ? & & \\
\hline on ne sait pas encore exactement & $\mathbf{5 5}$ & 34,5 \\
\hline en lui inventant une histoire & 15 & $\mathbf{7 3 , 5}$ \\
\hline en lui racontant comment ça s'est passé & $\mathbf{7 2 , 5}$ & 16 \\
\hline
\end{tabular}

\section{Le don, ses spécificités, ses principes}

Un ensemble de questions visait à saisir le sens donné au don de sperme, en comparaison aux autres formes de don existant. Ces figures de comparaison seront présentées par la suite accompagnées des principes qui définissent d'un 
point de vue législatif le don de sperme en France et au sujet desquels nous avons proposé différentes alternatives (tableau IV).

Tableau IV. - Qualifier le don.

\begin{tabular}{|l|c|c|}
\hline Diriez-vous que le don de sperme est... : & $\begin{array}{c}\text { pas d'accord } \\
\mathbf{\%}\end{array}$ & $\begin{array}{c}\text { d'accord } \\
\mathbf{\%}\end{array}$ \\
\hline un don comme un autre & 44,9 & $\mathbf{5 3 , 6}$ \\
\hline un don particulier, car il transmet l'ADN & 33,7 & $\mathbf{6 3 , 9}$ \\
\hline un geste presque héroïque & $\mathbf{5 2 , 3}$ & 44 \\
\hline un acte généreux et désintéressé comme un autre & 16,7 & $\mathbf{8 1 , 4}$ \\
\hline un acte médical comme un autre & $\mathbf{5 9 , 9}$ & 37,9 \\
\hline un prélèvement & $\mathbf{5 4 , 8}$ & 41,7 \\
\hline
\end{tabular}

Le don tire ses spécificités de par ses propriétés génétiques (transmission d'ADN, nécessitant un prélèvement et un contexte médical), tout en appartenant à la "famille » générale des dons (comme un autre, généreux). Oui, mais laquelle? (tableau V)

Tableau V. - Proximité du don.

\begin{tabular}{|l|c|c|}
\hline Le don de sperme est proche de celui... & $\begin{array}{c}\text { pas d'accord } \\
\%\end{array}$ & $\begin{array}{c}\text { d'accord } \\
\%\end{array}$ \\
\hline de sang & $\mathbf{5 0 , 6}$ & 46,5 \\
\hline d'ovule & 10,8 & $\mathbf{8 6 , 7}$ \\
\hline de moelle & 37,6 & $\mathbf{5 9}$ \\
\hline d'organe & 36,3 & $\mathbf{6 1 , 4}$ \\
\hline de plasma & 44,4 & $\mathbf{5 0 , 6}$ \\
\hline caritatif & $\mathbf{6 5 , 1}$ & 30,2 \\
\hline humanitaire & $\mathbf{5 4 , 3}$ & 42,8 \\
\hline
\end{tabular}

C’est précisément la question traitée par la suite visant à définir les frontières de proximité. En tant que don de matériel génétique, le don de sperme est placé très proche de celui d'ovule, d'organe et de plasma. Paradoxalement, le don de sang n'est pas majoritairement associé au don de sperme, même si la référence à ce dernier est un constat permanent que nous avons fait aussi bien dans le discours des donneurs que dans celui des couples. Une explication possible pourrait être l'effet de halo produit par les différentes propositions du tableau. Les dons de nature humanitaire et caritative ont été écartés (tableau V).

Les couples demandeurs approuvent massivement $(92,1 \%)$ le choix de la formule législative actuelle pour le don de sperme, c'est-à-dire, anonyme et gratuit. Toutes les autres options proposées sont rejetées. Seule l'hypothèse de la non-gratuité du don (à condition que ce dernier reste anonyme) semble faire légèrement hésiter certains répondants, nous allons y revenir. Toutefois, il faut noter que lorsque la question du maintien du principe actuel de la gratuité est posée, l'approbation est également massive, à 89,6\% (tableau VI). 
Tableau VI. - Anonymat et gratuité.

\begin{tabular}{|c|c|c|}
\hline $\begin{array}{l}\text { Quelle est la meilleure formule à appliquer au don de } \\
\text { sperme? Qu'il soit : }\end{array}$ & $\begin{array}{c}\text { pas d'accord } \\
\%\end{array}$ & $\begin{array}{c}\text { d'accord } \\
\%\end{array}$ \\
\hline anonyme et gratuit & 5,1 & $\mathbf{9 2 , 1}$ \\
\hline non anonyme et gratuit & $\mathbf{7 9 , 1}$ & 12,5 \\
\hline anonyme et non gratuit & $\mathbf{7 0}$ & 22,2 \\
\hline non anonyme et non gratuit & $\mathbf{8 8 , 5}$ & 3,2 \\
\hline
\end{tabular}

La question suivante laissait le choix aux couples parmi cinq possibilités (non exclusives) pour le donneur et le don faisant ainsi écho aux registres d'information sur les donneurs existant dans d'autres législations nationales. Force est de constater que hormis le cas de figure des informations non identifiantes pour le donneur, le choix des couples a majoritairement porté sur la conservation du système actuel excluant la possibilité d'un accès pour les parents ou pour l'enfant à naître dès sa majorité à des informations identifiantes pour le donneur (tableau VII).

Tableau VII. - Cinq possibilités pour le donneur.

\begin{tabular}{|l|c|c|}
\hline $\begin{array}{l}\text { À supposer que vous puissiez choisir entre } \\
\text { ces cinq possibilités pour le donneur, } \\
\text { laquelle choisiriez-vous? }\end{array}$ & $\begin{array}{c}\text { pas d'accord } \\
\%\end{array}$ & $\begin{array}{c}\text { d'accord } \\
\%\end{array}$ \\
\hline $\begin{array}{l}\text { Un donneur anonyme pour lequel aucune information } \\
\text { n'est et ne sera disponible }\end{array}$ & 26,8 & $\mathbf{6 8 , 5}$ \\
\hline $\begin{array}{l}\text { Un donneur pour lequel des informations } \\
\text { non identifiantes sont disponibles }\end{array}$ & 38,3 & $\mathbf{5 6}$ \\
\hline $\begin{array}{l}\text { Un donneur dont l'identité est conservée et qui peut être } \\
\text { connu à la demande des parents }\end{array}$ & $\mathbf{7 3}$ & 20,2 \\
\hline $\begin{array}{l}\text { Un donneur dont l'identité est conservée et qui peut être } \\
\text { connu à la demande de l'enfant à sa majorité }\end{array}$ & $\mathbf{7 3 , 5}$ & 21,9 \\
\hline $\begin{array}{l}\text { Un donneur dont l'identité est conservée et qui } \\
\text { peut être connu à la demande des parents et de l'enfant } \\
\text { à sa majorité }\end{array}$ & $\mathbf{7 3 , 2}$ & 21,6 \\
\hline
\end{tabular}

Les réponses des couples confirment les résultats de la recherche qualitative sur la question de la gratuité. Ainsi, ils considèrent majoritairement que la rémunération pourrait attirer davantage de donneurs potentiels, tout en reconnaissant que cela altérerait la nature "bénévole » du don. La rémunération est clairement une question qui divise, étant donné que 53,3\% de sujets indiquent qu'ils ne s'y opposeraient pas à condition de respecter un choix médicalement rigoureux de donneurs. Cependant, deux tiers de couples $(69,8 \%)$ déclarent que cette disposition remettrait en cause leur propre démarche (tableau VIII). 
Tableau VIII. - Formule à appliquer au don.

\begin{tabular}{|l|c|c|}
\hline $\begin{array}{l}\text { Quelle est la meilleure formule à appliquer au don } \\
\text { de sperme? }\end{array}$ & $\begin{array}{c}\text { pas d'accord } \\
\%\end{array}$ & $\begin{array}{c}\text { d'accord } \\
\%\end{array}$ \\
\hline $\begin{array}{l}\text { Si le don de sperme était rémunéré, il y aurait plus de } \\
\text { donneurs }\end{array}$ & 23,5 & $\mathbf{7 4 , 4}$ \\
\hline $\begin{array}{l}\text { Si le don de sperme n'est plus gratuit, les gens feront ça } \\
\text { d'abord pour l'argent }\end{array}$ & 21,4 & $\mathbf{7 5 , 9}$ \\
\hline Si le don n'est plus gratuit, ça n'est plus un don & 20,9 & $\mathbf{7 7 , 1}$ \\
\hline $\begin{array}{l}\text { C'est parce que le don est gratuit qu'on peut faire confiance } \\
\text { aux donneurs }\end{array}$ & 27,3 & $\mathbf{6 9 , 8}$ \\
\hline $\begin{array}{l}\text { Je serai favorable à la rémunération des donneurs à condi- } \\
\text { tion qu'ils soient sélectionnés de manière rigoureuse }\end{array}$ & $\mathbf{5 3 , 3}$ & 43,8 \\
\hline $\begin{array}{l}\text { Je n'aurais pas procédé à une démarche d'IAD si le don était } \\
\text { rémunéré pour les donneurs }\end{array}$ & $\mathbf{6 9 , 8}$ & 23,6 \\
\hline
\end{tabular}

\section{Divergences législatives, convergences parentales}

Une lecture croisée des nombreux rapports et recommandations officiels produits ces derniers mois en France laissent à penser que les lois françaises de bioéthique se trouveront modifiées dès 2010. En effet, si nous nous focalisons uniquement sur le don de sperme, de nombreux avis émis prônent, à des degrés différents, une ouverture relative du statut actuel de l'anonymat du donneur de sperme [12]. Le débat sous-jacent à cette orientation du changement des lois vers un anonymat plus souple oscille entre, d'un côté, le droit de l'enfant d'avoir accès à ses origines (et si oui, jusqu'à quel degré), de l'autre, le droit et l'intérêt du donneur à rester anonyme, et mentionne, enfin, le souci du maintien d'un équilibre familial. Les tenants de l'anonymat pensent que l'information sur les origines pourrait être dommageable pour l'enfant et pour les relations familiales; par ailleurs, une crainte de la baisse drastique des donneurs est évoquée comme conséquence première d'une éventuelle levée totale ou partielle de l'anonymat. À l'inverse, les défenseurs de l'accès à l'information sur les origines clament les conséquences psychosociales préjudiciables à l'enfant dues au secret en se référant notamment aux études concernant les familles adoptives et à la littérature sur les thérapies familiales. Ces dernières années, ce débat est porté sur l'espace public et médiatique français aussi bien par un certain discours «psy » basé sur la preuve clinique des conséquences psychiques néfastes constatées auprès de cas d'enfants nés grâce à un don de sperme (enfants IAD) venus en consultation, que par certains enfants IAD regroupés au sein d'associations témoignant et militant pour la levée totale de l'anonymat de ce type de don.

L'argumentation préconisant un changement radical de la loi, allant jusqu'à prôner la levée totale de l'anonymat pour les donneurs, se réfère, entre autres, par comparaison, aux pays ayant procédé depuis peu (Grande-Bretagne en 2005) ou depuis longtemps (Suède en 1985) à ce type de changement légis- 
latif. Néanmoins, dans les pays où une telle modification a eu lieu, la levée de l'anonymat n'a pas incité la majorité des parents à révéler à leurs enfants nés par don de sperme leurs conditions de conception. En effet, la divergence législative se traduit par une convergence des pratiques parentales dans le sens d'un maintien du secret vis-à-vis de l'enfant quant aux conditions de sa conception. Autrement dit, quelles que soient les possibilités offertes par le cadre législatif, les couples devenus parents grâce à l'IAD font davantage le choix du maintien du secret auprès de leurs enfants. À titre d'exemple, reprenons le cas de la Suède où la levée de l'anonymat date du milieu des années 1980. Concrètement, dès l'âge de 18 ans, un enfant né grâce à un don de sperme a la possibilité d'accéder à des informations identifiantes concernant le donneur. Cependant, l'impact de cette législation sur le plan des pratiques parentales reste insignifiant. Pour preuve, seulement 10 à $15 \%$ des parents concernés informent leurs enfants des conditions de leur conception $[10,13]$. Plusieurs raisons sont évoquées pour justifier ce choix par/pour les parents, citons principalement la protection des enfants, l'inquiétude de l'impact du récit sur l'équilibre relationnel de l'enfant avec le père ou la mère, ou encore, le maintien des relations sociales extrafamiliales.

Il convient d'ajouter que ne pas révéler n'équivaut pas forcément à se taire. «Un secret connu de deux personnes n'est déjà plus un secret » selon Simmel [14], et celui du mode de procréation ne fait pas exception à la règle. Aussi bien les couples interrogés dans notre étude que ceux de nombreuses études citées [15] parlent de leur démarche, partagent leur vécu, révèlent leur secret. L'entourage proche, le cercle familial (ou médical) leur sert d'interlocuteur confidentiel; ils prennent ainsi, consciemment ou inconsciemment, le risque d'une rupture future, accidentelle ou volontaire, de leur secret. Cependant, que cela soit en Suède, en Hollande, aux États-Unis, en Nouvelle Zélande ou encore en Espagne $[7,11,15,16]$, la convergence des pratiques parentales en matière de non-révélation du récit de conception est frappante.

Dans nos propres résultats, les couples demandeurs privilégient massivement le choix de la formule législative actuelle en France pour le don de sperme, c'est-à-dire, anonyme et gratuit. Toutes les autres options proposées ont été rejetées. En ce qui concerne les informations que les couples souhaiteraient obtenir quant au donneur, force est de constater que, hormis le cas de figure des informations non identifiantes sur le donneur, le choix des couples a également majoritairement porté sur la conservation du système actuel. Ce choix exclut la possibilité d'un accès, pour les parents ou pour l'enfant à naître à sa majorité, à des informations identifiantes sur le donneur. Ainsi, les couples restent attachés au modèle et aux règles actuelles de droit qui régissent la démarche dans laquelle ils se trouvent, pour la grande majorité d'entre eux, pour la première fois. Seule l'option d'une disponibilité d'informations non identifiantes sur le donneur recueille environ la moitié des avis favorables.

Néanmoins, au moment de la passation des questionnaires, c'est-à-dire à un moment où les couples sont présents aux CECOS pour l'élaboration de leur demande de don, les réponses en termes d'intention de parler à l'enfant 
sont clairement et majoritairement positives et équilibrées par genre. Notre échantillon de couples témoigne donc d'un potentiel conséquent d'intentions déclarées d'en parler, deux tiers de répondants excluant de "ne rien dire » à l'enfant. Toutefois, rappelons ici les spécificités du contexte de notre recherche impliquant l'interrogation de couples pris dans la temporalité subjective et institutionnelle de leur démarche et dans un désir et une attente intenses de procréation, ce qui ne nous permet pas de risquer des comparaisons. Rappelons également qu'à l'heure actuelle, le suivi longitudinal de couples ayant fondé leur famille grâce à l'IAD en France est très délicat, voire impossible.

La décision des couples de ne pas révéler les conditions de conception à leur enfant correspond avant tout à une stratégie identitaire de protection et traduit une forme de vulnérabilité parentale. Protection contre des réactions négatives possibles issues du cercle social, familial et amical, cette stratégie vise aussi bien à protéger les parents, principalement le père, que l'enfant. Une possible mise en cause de l'identité de l'homme sous l'angle de la parentalité (sa qualité d'être père) et de la masculinité (sa qualité d'être homme), de la force des liens qui l'unissent à l'enfant, renforce la crainte d'un double stigmate, social et identitaire. Sans négliger l'expérience souvent traumatisante de l'annonce de la stérilité masculine. Le bien-être de l'enfant, son équilibre, le constat d'une vie familiale équilibrée complètent le tableau des argumentaires prônant ou plutôt justifiant cette posture. À l'inverse, le droit de l'enfant de savoir, la peur de l'apprendre par quelqu'un d'extérieur à la famille et le risque d'endogamie consanguine, sont évoqués comme raisons principales par la minorité des familles ayant parlé à leurs enfants de l'IAD. Ces stratégies témoignent des pôles de tension, éthiques et identitaires, psychologiques et sociétaux qui se situent sur un continuum entre ce que les parents peuvent/savent/doivent dire et ce que l'enfant doit/peut/veut savoir.

L'anonymat et le secret constituent empiriquement les principes organisateurs essentiels qui structurent les prises de position des couples impliqués dans le don de sperme en tant que demandeurs-receveurs. Cependant, ces deux principes ne suffisent pas à eux seuls à rendre compte de la diversité des prises de position sur des questions particulières. Le rapport au donneur, sous l'angle de l'intérêt, de la dépersonnalisation ou encore de l'éventualité de son "retour », ainsi que les enjeux liés à la transmission et à la ressemblance contribuent eux aussi à structurer ce champ représentationnel. C'est donc au sein d'un réseau de représentations sociales [17], large et complexe, qu'est vécue la démarche de l'IAD, que sont fait les choix de parler aux autres ou à l'enfant, et que se déterminent des positionnements sur l'anonymat, le secret ou la gratuité. 


\section{Remerciements}

Cette recherche a été réalisée grâce à un financement de l'agence de la biomédecine ( $\left.\mathrm{n}^{\mathrm{O}} 2006-901\right)$ région Rhône-Alpes ( $\left.\mathrm{n}^{\mathrm{O}} 0602279801-\mathrm{TZ014}\right)$ attribué à Nikos Kalampalikis. Nous sommes reconnaissants envers la Fédération française des CECOS pour le partenariat de recherche fructueux. Nos sincères remerciements sont adressés aux participants qui ont accepté de partager une part de leur vécu.

\section{Références}

1. Hennebicq S, Fédération française des CECOS (2004) Trente années de Cecos : 30 années de bilans. La Tribune des Cecos 15:12-5

2. Kalampalikis N, Haas V, Fieulaine N, et al. (2009) Enjeux éthiques et identitaires dans l'acte de procréation par don de sperme. Rapport de fin de recherche, Agence de la biomédecine et région Rhône-Alpes, Groupe de recherche en psychologie sociale (GRePS EA 4163) Lyon-II

3. Flick W (1992) Triangulation revisited: strategy of validation or alternative? J Theory Soc Behav 22:175-97

4. Cadoret A, Wilgaux J (2007) Nécessaire anonymat et secret de soi. Réflexions sur les lois bioéthiques, Ethnol Fr XXXVII: 135-42

5. Caillé A (2000) Anthropologie du don. Desclée de Brouwer, Paris

6. Godelier M (2004) Métamorphoses de la parenté. Fayard, Paris

7. Brewaeys A, Golombok S, Naaktgeboren N, et al. (1997) Donor insemination: Dutch parents' opinions about confidentiality and donor anonymity and the emotional adjustment of their children. Hum Reprod 12:1591-7

8. Hunter M, Salter-Ling N, Glover L (2000) Donor insemination: telling children about their origins. Child Psychol Psychiatry Review 5:157-63

9. Rumball A, Adair V (1999) Telling the story: parents' scripts for donor offspring. Hum Reprod 14:1392-9

10. van Berkel D, van der Veen L, Kimmel I, te Velde E (1999) Differences in the attitudes of couples whose children were conceived through artificial insemination by donor in 1980 and in 1996. Fertil Steril 71:226-31

11. Grace VM, Daniels KR, Gillett W (2008) The donor, the father and the imaginary constitution of the family: parents' constructions in the case of donor insemination. Soc Sci Med 66:301-14

12. Claeys A, Vialatte JS (2008) Rapport sur l'évaluation de l'application de la loi $\mathrm{n}^{\mathrm{o}} 2004-$ 800 du 6 août 2004 relative à la bioéthique. Office parlementaire d'évaluation des choix scientifiques et technologiques. Assemblée nationale et sénat

13. Gottlieb C, Lalos O, Lindblad F (2000) Disclosure of donor insemination to the child: the impact of Swedish legislation on couples' attitudes. Hum Reprod 15:2052-6

14. Simmel G (1996) Secret et sociétés secrètes. Circé, Paris

15. Golombok S, Brewaeys A, Giavazzi MT, et al. (2002) The European study of assisted reproduction families: the transition to adolescence. Hum Reprod 17:830-40

16. Nachtigall RD, Becker G, Quiroga SS, Tschann JM (1998) The disclosure decision: concerns and issues of parents of children conceived through donor insemination. Am J Obstet Gynecol 178:1165-70

17. Kalampalikis N, Haas V (2008) More than a theory: a new map of social thought. J Theory Soc Behav 38:449-59 